\title{
Modeling of Communication Delays Aiming at the Design of Networked Supervisory and Control Systems. A First Approach
}

\author{
Karina Cantillo ${ }^{1}$, Rodolfo E. Haber ${ }^{1}$, Angel Alique ${ }^{1}$, and Ramón Galán ${ }^{2}$ \\ ${ }^{1}$ Instituto de Automática Industrial - CSIC, Campo Real km 0.200, \\ Arganda del Rey, Madrid 28500 \\ \{cantillo, rhaber, a.alique\}@iai.csic.es \\ ${ }^{2}$ Escuela Técnica Superior de Ingenieros Industriales. Universidad Politécnica de Madrid, \\ Calle José Gutiérrez Abascal, nº 2, Madrid 28006 \\ rgalan@etsii.upm.es
}

\begin{abstract}
This paper presents a first approach to model the communication delays with the aim of improving the design of networked supervisory and control systems (NSCS). Network delays affect the performance of NSCS. Therefore, it is necessary a complete analysis, modeling and simulation of the communication delays upon the connection technology in use (e.g., Ethernet networks). A statistical model is proposed on the basis of autocorrelation of LRD that is imposed by the bursty behavior of the network traffic.
\end{abstract}

\section{Introduction}

Nowadays, data networks (i.e., Ethernet networks) have become in a low-cost connection media for networked supervisory and control systems (NSCS) [1], due mainly to the improvement concerning with the speed of communication and the bandwidth. It is evident the need to perform the analysis, modeling and simulation of the communication delays in the framework of NSCS. A suitable and reliable model of communication delays allows assessing the network behavior, the overall influence of the delays on the deterioration of the NSCS performance and the sufficient conditions for closed-loop stability.

In the next section is detailed the developed framework to measure communication delays. The developed model is shown in section 3. In subsection 3.2 is presented the communication delays simulator. Finally, some conclusions and remarks are given.

\section{Communication Delays Measurement over the Propose Work Environment}

The case of study consists of two applications based on RT-CORBA for measuring delays samples. Applications are executed using TCP/IP protocol over an Ethernet network with 110 stations and $10 \mathrm{Mbs}$ of bandwidth. Real-Time CORBA defines 
mechanisms and policies to control processor, communication and memory resources. The ACE ORB, unlike the most of CORBA implementations, shares a minimum part of ORB resources, reducing substantially the synchronization costs and the priority inversion between the process threads, offering a predictable behavior [2]. The communication interface defined for the applications is depicted below.

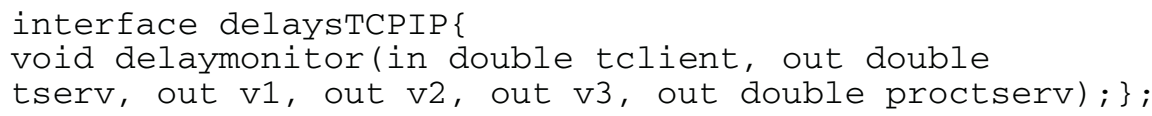

\section{Data Network Delays Models: The Use of Statistical Techniques}

Some network traffic models well-described in the literature are inspired in statistics method such as Poisson Processes, Markov chain, time series, heavy-tailed models, self-similar process and others. These models can be considered as black box tools able to link cause-effect and emulate network traffic behavior with a high accuracy.

The bursty behavior of the Ethernet technology in network traffic imposed the inclusion of the autocorrelation concept in the model to be defined. Poisson processes can be used for modeling the delay between continuous packets with the Exponential Distribution Function (EDF). The drawback is that these processes do not take into account the auto-correlation concept due to the independence hypothesis in data, leading erroneous simulations and analysis. In order to verify these constraints, a statistic analysis of data was performed with Quantile-Quantile graphic and the validation methods Chi-Square Test, Anderson-Darling Test. The results showed that $\mathrm{EDF}$ is not adequate to represent the samples of delay included in the measured data.

The introduction of the auto-correlation concepts in the model was then required. Initials solutions were simple chain Markov models, adjusted to a correlation of shortrange dependence (SRD), characterizing just in a minimal proportion the bursty behavior. In order to reach a whole representation, auto-correlation of long-range dependence (LRD) must be considered. In order to represent the LRD autocorrelation indices the self-similar processes are used, because the LRD can be characterized only with a simple parameter, the Hurst coefficient $(\mathrm{H})$.

\subsection{Inclusion of Self-similar Processes in the Proposed Model}

Self-similarity processes consist of repeated patterns in multiples scales. The literature shows that the network traffic is self-similar [3]. There are different methods for the Hurst coefficient estimation. The R/S method was chosen because it is easy to implement and it is very useful in other research fields. Initially, the developed program in Matlab makes use of a sample taken in a time-period with low network load. The estimated $H$ was 0.6535 . Subsequently, a sample taken in presence of higher network load was assessed, obtaining an estimated of $H=0.7232$. The results show how $H$ can be used as a reliable measure parameter of the bursty behavior in the network traffic. 
In order to complete the representation, a distribution function that fits the LRD characteristics must be chosen as the empiric distribution indicated by the samples. One solution is the use of processes with infinite variance called heavy-tail processes. The distribution functions Pareto, Weibull, Exponencial, Gamma, and Lognormal, were analyzed and compared using the central limit theorem and the infinite variance concept. Figure 1 shows the Log-Log plot of each one. The graphic shows that Pareto is the distribution function that better fits data behavior. A residual variance analysis was performed supporting previous result. Additionally, the Pareto distribution function is heavy-tail type that is recommended to depict the network delays.

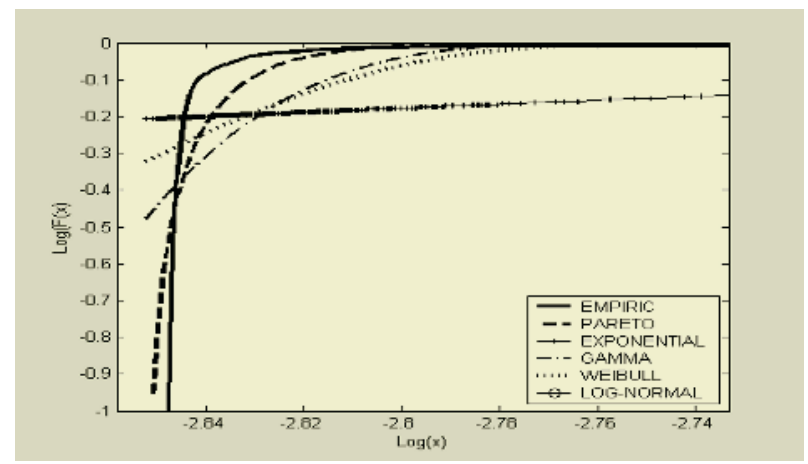

Fig. 1. Comparative Analysis: Empiric Cumulative Distribution Function (CDF) and Studied Cumulative Distribution Functions (Pareto, Exponential, Weibull, Gamma, Log-Normal)

\subsection{Communication Delays Generator}

The method Fractional Gaussian Noise (FGN) was chosen to generate pure fractal signals based on the Hurst parameter, due to its implementation easiness [4]. The developed simulator initially calculates the Hurst coefficient, based in the R/S method. This coefficient is used to generate the self-similar sample. The sample is mapped to the Pareto distribution function and then formatted to obtain a simulation close to the reality. Before the formatted procedure, the equation used is $Y_{i}=\left(\frac{a \times b}{\log \left(1-F_{n}\left(X_{i}\right)\right)}\right) \cdot F_{n}$ is the normal cumulative distribution function, $a$ is the shape parameter, $b$ is the minimum value of the analyzed sample and $X_{i}$ is the datum obtained by FGN method. Figure 2 shows a comparison in a log-log plot between the empiric cumulative distribution of the analyzed sample and the empiric cumulative distribution of the sample obtained from the simulator.

In order to validate the results a residual variance analysis, between the sample generated by the simulator and a sample derived from a Poisson model, was accomplished. The developed simulator achieved the best representation. Although the defined model does not represent all the properties of networks traffic, as SRD behavior, this model is essential to assess the influence of the network delays in reliability and feasibility of NSCS design. 


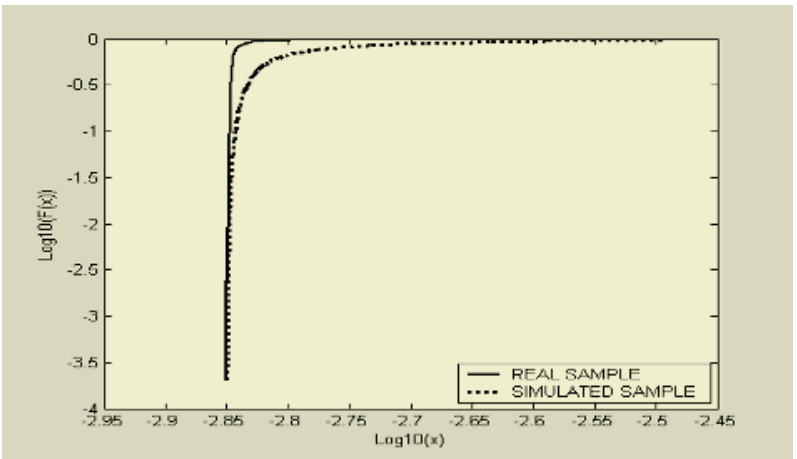

Fig. 2. Comparative Analysis: Real Sample and Simulated Sample

\section{Conclusions}

This paper proposes a statistical model. The bursty behavior of the network traffic imposed the use of self-similar processes using the Hurst coefficient. The results show how $H$ can be used as a performance index of the bursty behavior in the network traffic. Likewise, Pareto distribution function shows the best performance in representing the empiric distribution function derived from samples measured in realtime. On the other hand, this paper proposes a communication delays simulator using Fractional Gaussian Noise method. Both model and simulator are very useful to design networked supervisory and control systems although they do not take into account the autocorrelation of SRD in the case study.

\section{References}

1. Branicky, M.S., Phillips, S.M., Zhang, W.: Stability of Networked Control System: Explicit Analysis of Delay. Proceedings of the American Control Conference (2000) 2352-2357

2. Schmidt, D.C., Mungee, S., Gaitan, S.F, Gokhale, A.: Software Architectures for Reducing Priority Inversion and Non-determinism in Real-time Object Request Brokers. Journal of Real-time Systems, Vol. 21, 1-2, (2001) 77-125

3. Leland, W.E., Taqqu, M.S., Willinger, W., Wilson, D.V.: On the self-similar nature of Ethernet traffic. IEEE/ACM Transactions on Networking, Vol. 2 (1994) 1-15

4. Paxson, V.: Fast, Approximate Synthesis of Fractional Gaussian Noise for Generating SelfSimilar Network Traffic. ACM SIGCOMM CCR, Vol. 27 (1997) 5-18

5. Cantillo, K., Haber, R.E., Alique, A., Galan, R.: CORBA-Based open platform for processes monitoring. An application to a complex electromechanical process. In: Bubak M., Albada G.D., Sloot P.M.A., Dongarra J. (eds.). Computational Science ICCS2004, Lecture Notes in Computer Science 3036 (2004) 531-535 